\title{
Description of a Cognitive Radio Testbed based on USRP Platforms and CogWave
}

\author{
Vincent Le Nir and Bart Scheers \\ Royal Military Academy, Dept. CISS \\ 30, Avenue de la Renaissance \\ B-1000 Brussels BELGIUM.
}

\begin{abstract}
Today, many software defined radio (SDR) platforms are commercially available as off-the-shelf products at a very reasonable price. The concept of cognitive radio (CR) is to have a network of SDRs which can autoconfigure and autonomously change its parameters (waveform, frequency, bandwidth, power) according to the user needs and the electromagnetic environment. CogWave is an open-source software framework aiming at developing CR waveforms. Multiple waveforms have been implemented in CogWave, such as the multichannel DAA-OFDM waveform, the DADS waveform, and GNU Radio waveforms. CogWave provides the means to implement different $C R$ waveforms and to develop rules to switch between different $\mathrm{CR}$ waveforms during run-time according to the user needs and the electromagnetic environment. A description of a CR testbed based on USRP platforms and the CogWave framework is given. In the scenario, a jammer perturbs the data transmission between two CR nodes. Results show that when the throughput drops under a predifined threshold, the CR nodes are able to switch from one waveform to another waveform while maintaining communication.
\end{abstract}

\section{INTRODUCTION}

The aim of software defined radio (SDR) is to replace special analog hardware components by field programmable gate arrays (FPGA), digital signal processors (DSP) and general purpose processors (GPP) to allow fast-prototyping and to support multiple radio standards on a single reconfigurable platform [1]. Today, many SDR platforms are commercially available as off-the-shelf products at a very reasonable price. The concept of cognitive radio (CR) is to have a network of SDRs which can autoconfigure and autonomously change its parameters (waveform, frequency, bandwidth, power) according to the user needs and the electromagnetic environment [2]. CogWave is an open-source software framework aiming at developing CR waveforms [3]. CogWave uses the Qt framework, the communications library IT++, the USRP universal hardware driver (UHD) and other librairies to enable realtime transmission between USRP devices. Multiple waveforms have been implemented in CogWave. The first waveform is the multichannel orthogonal frequency division multiplexing (OFDM) waveform with detection and avoid (DAA) introduced in [4]. The second waveform is the delay and add direct sequence (DADS) waveform with a short spreading sequence introduced in [5]. Other waveforms such as OFDM, BPSK, QPSK, GMSK, CPFSK etc. have been ported to CogWave from GNU Radio [6]. A CR node is composed of a host PC running the CogWave framework and a USRP. CogWave gives the ability to the CR nodes to modify the waveform and its parameters during run-time according to the user needs and the electromagnetic environment. This paper gives a description a CR testbed based on USRP platforms and the CogWave framework with its two waveforms, i.e. the multichannel DAA-OFDM waveform and the DADS waveform. In the scenario, a jammer perturbs the data transmission between two CR nodes. Results show that when the throughput drops under a predefined threshold, the CR nodes switch from the DADS to the multichannel DAA-OFDM automatically while maintaining communication. Section II provides a description of the CogWave framework, the multichannel DAA-OFDM waveform and the DADS waveform. A description of the CR testbed and results are given in Section III. Section IV concludes this paper.

\section{Description OF COgWAVE FRAMEWORK}

This Section provides a description of the CogWave framework. The main objective of $\operatorname{Cog}$ Wave is to develop a library of waveforms and to develop rules to switch between these waveforms during run-time according to the user needs and the electromagnetic environment. Multiple waveforms have been implemented in CogWave. The first waveform is the multichannel DAA-OFDM waveform introduced in [4]. The multichannel DAA-OFDM waveform uses energy-based sensing to adjust dynamically the channel and to mitigate interference. It has no pilot symbols and requires blind time, frequency and phase recovery. The second waveform is the DADS waveform with a short spreading sequence introduced in [5]. The DADS waveform has a very low complexity and does not require frequency and phase recovery. Other waveforms such as OFDM, BPSK, QPSK, GMSK, CPFSK etc. have been ported from GNU Radio [6]. Either a frequency division duplex (FDD) or a time division duplex (TDD) mode can be used for all waveforms. In a TDD mode, a frame is composed of two slots to transmit and receive packets on the same frequency. In a FDD, mode, a frame is composed of a single slot to transmit and receive packets on different frequencies. Frequency hopping can be enabled by a predefined hopping sequence only known to the CR nodes. The USRP RF frontend allows to perform frequency hopping over a large frequency range from $70 \mathrm{MHz}$ to $6 \mathrm{GHz}$.

A slot has a fixed duration in order to carry a modulated data packet of variable size as described in Figure 1. Modulated 


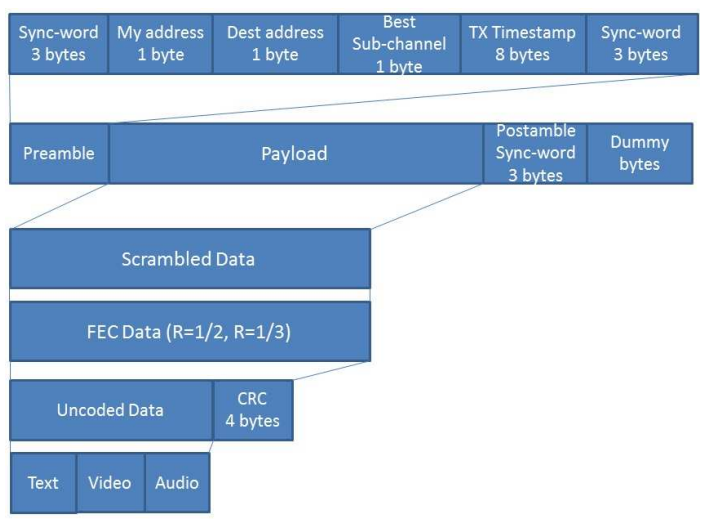

Fig. 1. Data packet in CogWave

dummy bytes are used to fill the slot period. Frame synchronization uses sync-words. The uncoded data is processed by cyclic redundancy check (CRC), forward error correction (FEC) and linear feedback shift register (LFSR) scrambling. This uncoded data can be received and transmitted to virtual network interfaces (TUN/TAP) to establish connections between $\mathrm{CR}$ nodes. This uncoded data can also be received and transmitted to named pipes for simple text messaging, video and audio streaming, or even in the case of hardwarein-the-loop network simulation/emulation/testbed with more complex frameworks such as OMNeT++ and ns-3 [7], [8]. Such frameworks allow to create CR nodes with a complete protocol stack from the MAC up to the application layer independently of the CogWave PHY layer.

\section{A. Description of the multichannel DAA-OFDM waveform}

The multichannel DAA-OFDM waveform has been introduced in [4]. The band of interest $B$ is composed of $N$ OFDM sub-carriers and is divided into a number of sub-channels $S$ of width $B / S$. Each channel is composed of $N / S$ OFDM subcarriers. A CR node determines which channel has the lowest energy every frame's period by

$$
S_{\text {opt }}=\min _{m} \sum_{i=\frac{m N}{S}}^{\frac{(m+1) N}{S}-1} E(i)
$$

with $m=[0, \ldots, S-1]$ in which $E(i)$ is the averaged periodogram of sub-carrier $i$ given by

$$
E(i)=\frac{1}{K N} \sum_{k=0}^{K-1}\left|\sum_{n=0}^{N-1} y(k N+n) e^{-\frac{j 2 \pi i n}{N}}\right|^{2}
$$

with $i=[0, \ldots, N-1]$ sub-carriers in which $y(k N+n)$ with $k=[0, \ldots, K-1], n=[0, \ldots, N-1]$ are the received complex samples for a slot period in the band of interest. A CR node OFDM-modulates the bit sequence of the slot on the channel which has the lowest energy. In a TDD mode, if a packet was received correctly on another channel, the same channel is used to OFDM-modulate the bit sequence. A CR node performs an OFDM signal detection and time recovery by the cyclic prefix based sliding window metric [9] given by

$\rho(\theta)=\frac{\left|\sum_{k=0}^{K-2} \sum_{j=\theta}^{\theta+P-1} y(k(N+P)+j) y^{*}(k(N+P)+j+N)\right|}{(K-1) P \sigma_{y}^{2}}$

with $P$ the cyclic prefix size. The time offset estimate is given by

$$
\theta^{o p t}=\max _{\theta \in\{0, \ldots, N+P-1\}} \rho(\theta)
$$

In order to allow the detection of an OFDM signal even in the presence of an unknown signal, the CR node uses a detection threshold based on the non-correlated part of the cyclic prefix based sliding window metric at the time offset estimate instead of the noise variance [4]. The non-correlated part of the cyclic prefix based sliding window metric at the time offset estimate is given by the vector $\left[\rho\left(\bmod \left(\theta^{\text {opt }}+\right.\right.\right.$ $\left.2 P, N+P)), \ldots, \rho\left(\bmod \left(\theta^{o p t}+N-P-1, N+P\right)\right)\right]$. Assuming that the non-correlated part is a Gaussian distribution with mean $m_{n c}$ and variance $\sigma_{n c}^{2}$, the detection threshold $\eta$ is given by

$$
\eta=m_{n c}+\alpha \sigma_{n c}
$$

with $\alpha$ an integer corresponding to the number of standard deviations necessary to discriminate between the cyclic prefix based sliding window metric at the timing offset estimate $\rho\left(\theta^{\text {opt }}\right)$ and its non-correlated part $\left[\rho\left(\bmod \left(\theta^{\text {opt }}+2 P, N+\right.\right.\right.$ $\left.P)), \ldots, \rho\left(\bmod \left(\theta^{o p t}+N-P-1, N+P\right)\right)\right]$. The detector scheme is

$$
\begin{array}{ll}
\rho\left(\theta^{\text {opt }}\right)>\eta & \text { Presence of an OFDM signal } \\
\rho\left(\theta^{\text {opt }}\right)<\eta & \text { Absence of an OFDM signal }
\end{array}
$$

The CR node also use the cyclic prefix based sliding window time offset estimate to estimate the frequency offset $\left(\epsilon^{o p t}\right)$ for carrier frequency recovery [10]

$$
\epsilon^{o p t}=-\frac{1}{2 \pi N} \angle \rho\left(\theta^{o p t}\right)
$$

After time and frequency offset corrections, the OFDM symbols are transformed to the frequency domain by the discrete Fourier transform (DFT) operation. As there is no interference between two consecutive OFDM symbols, independent sub-carriers are obtained with the following channel model

$$
Y(k N+i)=H(k N+i) X(k N+i)+N(k N+i)
$$

with $k=[0, \ldots, K-1]$ and $i=[0, \ldots, N-1]$, in which $Y(k N+i), H(k N+i), X(k N+i)$, and $N(k N+i)$ are respectively the demodulated data, the channel frequency response, the transmitted symbol and the noise for the block $k$ and sub-carrier $i$. Assuming the channel amplitude invariant over the $K$ blocks, a blind estimate of the channel amplitude for automatic gain control is given by 


$$
\left|H(i)^{o p t}\right|^{2}=\frac{1}{K} \sum_{k=0}^{K-1}|Y(k N+i)|^{2}
$$

The channel phase varies between sub-carriers due to an incorrect timing offset estimate and the channel frequency response. The channel phase also varies between blocks in time due to a remaining frequency offset or oscillator phase noise. The two-dimensional carrier phase recovery given by Algorithm 1 uses the second-power estimator for the BPSK constellation $(M=2)$ and the fourth-power estimator for QAM constellations $(M=4)$ [11], [12]. A 1-D phase unwrapping is performed in the frequency domain to correct linear shifts of the phase in the frequency domain due to an incorrect timing offset as well as abrupt changes of the phase due to the phase ambiguity of the $M$-power estimators. Another 1-D phase unwrapping is performed in the time domain to correct a remaining frequency offset or oscillator phase noise. The $M$-fold phase ambiguity is mitigated using sync-words in the packet preamble.

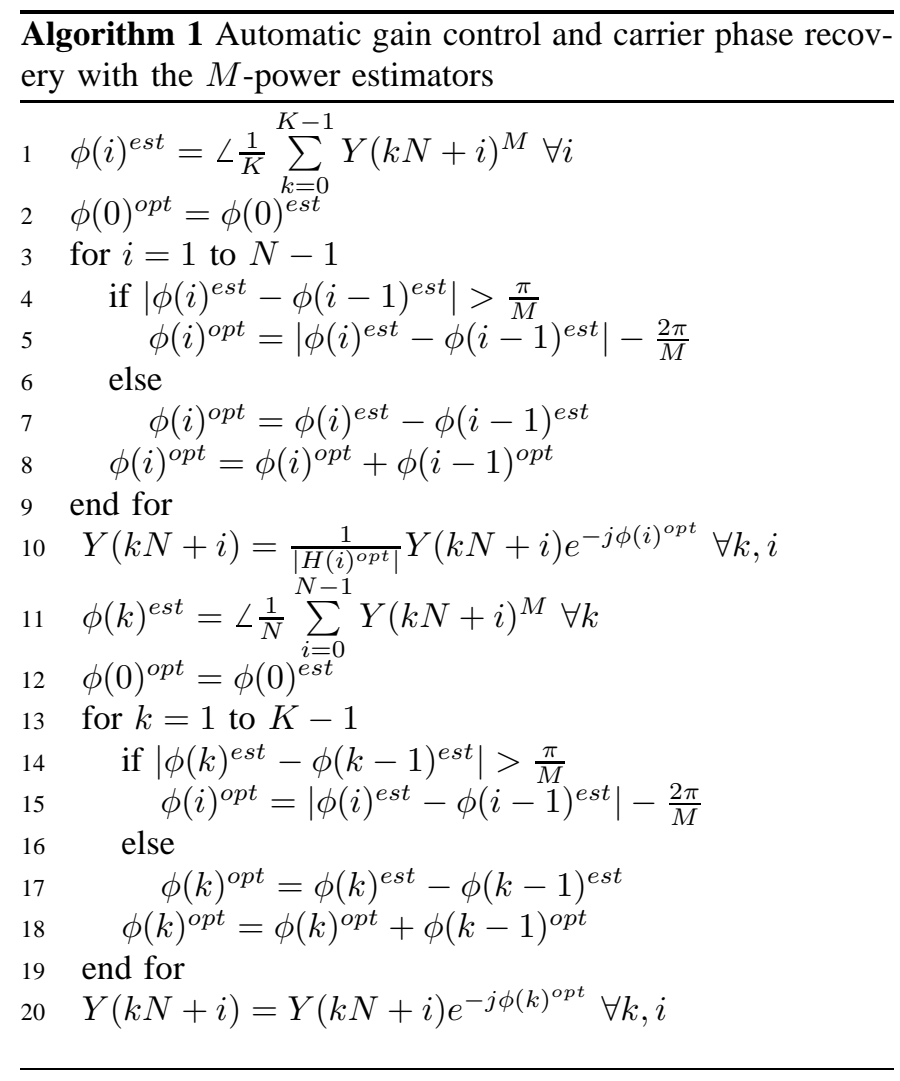

\section{B. Description of the DADS waveform}

The DADS waveform has been introduced in [5]. A CR node modulates the bit sequence of the slot by the transmission chain shown in Figure 2. Assuming that $K$ bits have to be transmitted, the PN sequence of length $M$ is repeated $K$ times to form the reference signal. The transmitted signal is the sum of two signals, namely the reference signal and its delayed version multiplied by the information signal. The received signal $r_{i}$ can be modeled as

$$
r_{i}=\sum_{l=0}^{L-1} h_{l}\left(d_{k} x_{i-l-D}+x_{i-l}\right)+n_{i}
$$

with $D$ the delay (in chips), $d_{k}$ the information bits taking values in $\{-1,1\}$ with data rate $1 / M, x_{i}$ the transmitted chip of the PN sequence, $n_{i}$ the AWGN with variance $N_{0} / 2$ per dimension, $L$ the number of taps and $h_{l}$ the complex-valued channel attenuation for the $l^{\text {th }}$ tap. The selection of a short PN sequence whose length $M$ is twice the delay $D$ used in the modulation scheme $(M=2 D)$ is given by [5]

$$
\text { select }\left\{x_{i}\right\}=\left\{\begin{array}{l}
x_{i}=x_{i-2 D} \\
\sum_{i=D+1}^{M} x_{i} x_{i-D}=0
\end{array} \quad \forall i\right.
$$

A PN sequence satisfying this criterion can be easily generated from the $2^{M}$ possible codes. The ratio between the number of codes satisfying the auto-correlation criterion and the total number of codes $2^{M}$ for delays $D=2,4,6,8$ are 0.5 , $0.375,0.3125$, and 0.2734 respectively. The reception chain of the CR node is shown in Figure 3. The correlator output is given by

$$
S_{k}=\operatorname{Re}\left(\sum_{i=(k-1) M+D+1}^{k M} r_{i} r_{i-D}^{*}\right)
$$

with

$$
\begin{aligned}
& r_{i} r_{i-D}^{*}=\underbrace{d_{k} \sum_{l=0}^{L-1}\left|h_{l}\right|^{2}\left(x_{i-l-D}^{2}+x_{i-l}^{2}\right)}_{\text {useful part } a_{i}} \\
& +2 \sum_{l=0}^{L-1}\left|h_{l}\right|^{2} x_{i-l} x_{i-l-D} \\
& \underbrace{+\sum_{l=0}^{L-1} \sum_{l^{\prime} \neq l} h_{l} h_{l^{\prime}}^{*}\left(d_{k} x_{i-l-D}+x_{i-l}\right)\left(d_{k} x_{i-l^{\prime}}+x_{i-l^{\prime}-D}\right)}_{\text {interference part } b_{i}} . \\
& +\sum_{l=0}^{L-1} h_{l} n_{i-D}^{*}\left(d_{k} x_{i-l-D}+x_{i-l}\right) \\
& \underbrace{+\sum_{l=0}^{\substack{l=0 \\
L-1}} h_{l}^{*} n_{i}\left(d_{k} x_{i-l}+x_{i-l-D}\right)+n_{i} n_{i-D}^{*}} \\
& \text { noise part } c_{i}
\end{aligned}
$$

The correlator output can be divided into a useful, interference and noise parts

$$
\left\{A_{k}, B_{k}, C_{k}\right\}=\operatorname{Re}\left(\sum_{i=(k-1) M+D+1}^{k M}\left\{a_{i}, b_{i}, c_{i}\right\}\right) .
$$

Assuming that the correlator output approaches a Gaussian distribution, the bit error rate (BER) performance can be expressed semi-analytically as 


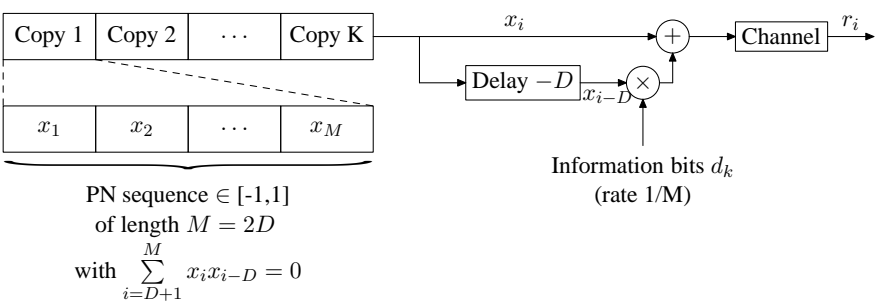

Fig. 2. Transmission chain of the DADS modulation scheme with the selection of a short PN sequence

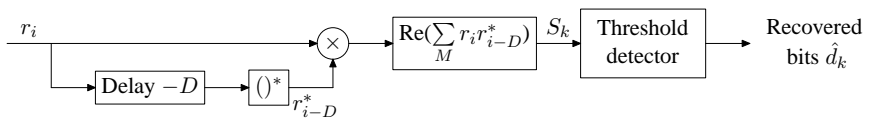

Fig. 3. Reception chain of the DADS modulation scheme

$$
\begin{aligned}
B E R= & \frac{1}{2} E_{h_{l}}\left[\operatorname{Prob}\left(S_{k}<0 \mid d_{k}=+1\right)\right. \\
& \left.+\operatorname{Prob}\left(S_{k} \geq 0 \mid d_{k}=-1\right)\right] \\
= & \frac{1}{4} E_{h_{l}}\left[\operatorname{erfc}\left(\frac{E\left[S_{k} \mid d_{k}=+1\right]}{\sqrt{2\left(\operatorname{var}\left[S_{k} \mid d_{k}=+1\right]\right)}}\right)\right. \\
& \left.+\frac{1}{4} \operatorname{erfc}\left(\frac{-E\left[S_{k} \mid d_{k}=-1\right]}{\sqrt{2\left(\operatorname{var}\left[S_{k} \mid d_{k}=-1\right]\right)}}\right)\right]
\end{aligned}
$$

with $\operatorname{erfc}($.$) the complementary error function. A_{k}$ and $B_{k}$ are deterministic values. For large $M$, the correlator output approaches a Gaussian distribution with mean and variance

$$
\begin{aligned}
& E\left[S_{k}\right]=A_{k}+E\left[B_{k}\right]+E\left[C_{k}\right] \\
& \operatorname{var}\left[S_{k}\right]=\operatorname{var}\left[B_{k}\right]+\operatorname{var}\left[C_{k}\right]
\end{aligned}
$$

The integrated useful part $A_{k}$, interference part $B_{k}$ and the mean of the noise part $C_{k}$ are given by

$$
\begin{array}{ll}
A_{k} & =2 d_{k} \sum_{l=0}^{L-1}\left|h_{l}\right|^{2}(M-D) P_{s} \\
E\left[B_{k}\right] & =0 \\
E\left[C_{k}\right] & =0
\end{array}
$$

with $P_{s}$ the energy per chip and the variance of the interference part $B_{k}$ and the variance of the noise part $C_{k}$ are given by

$$
\begin{aligned}
\operatorname{var}\left[B_{k}\right] & =4 \sum_{l=0}^{L-1}\left|h_{l}\right|^{4}(M-D) P_{s}^{2} \\
& +4 \sum_{l=0}^{L-1} \sum_{l^{\prime} \neq l}\left|h_{l}\right|^{2}\left|h_{l^{\prime}}\right|^{2}(M-D) P_{s}^{2} \\
\operatorname{var}\left[C_{k}\right] & =4 \sum_{l=0}^{L-1}\left|h_{l}\right|^{2}(M-D) P_{s} \frac{N_{0}}{2}+(M-D) \frac{N_{0}^{2}}{2}
\end{aligned}
$$

Knowing that a transmitted data bit is the sum of two sequences of length $M$, the energy per bit $E_{b}$ can be written

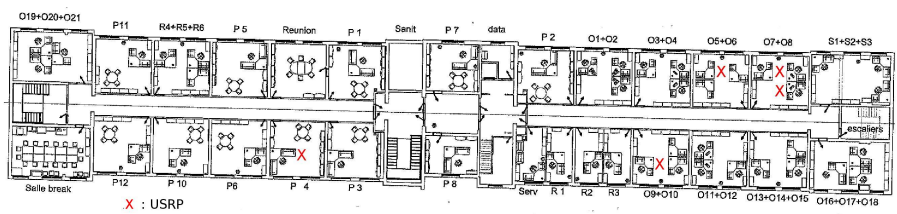

Fig. 4. Locations of the USRPs for the CR testbed

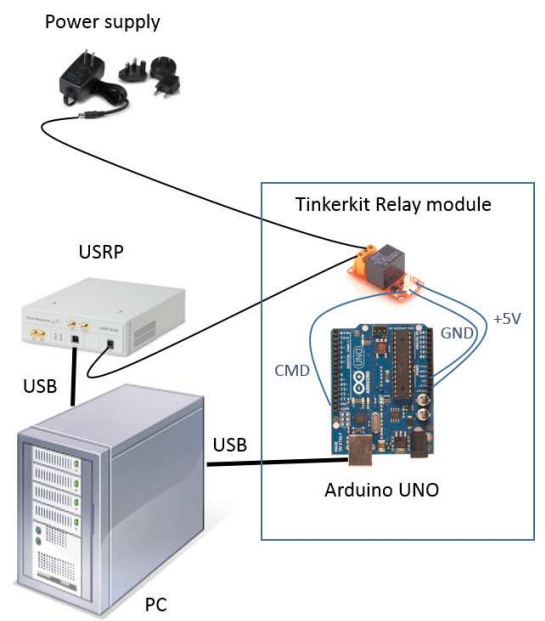

Fig. 5. Power switch for a USRP based on Arduino UNO and Tinkekit relay modules

as $E_{b}=2 M P_{s}$, the derivation of the BER formula leads to the following expression [5]

$$
B E R=\frac{1}{2} E_{h_{l}}\left[\operatorname{erfc}\left(\sqrt{\frac{\frac{M-D}{M} \sum_{l=0}^{L-1}\left|h_{l}\right|^{2} E_{b}}{2 N_{0} \Gamma}}\right)\right]
$$

with

$$
\Gamma=1+\frac{\sum_{l=0}^{L-1}\left|h_{l}\right|^{4} E_{b}}{\sum_{l=0}^{L-1}\left|h_{l}\right|^{2} M N_{0}}+\frac{M N_{0}}{2 \sum_{l=0}^{L-1}\left|h_{l}\right|^{2} E_{b}}+\frac{\sum_{l=0}^{L-1} \sum_{l^{\prime} \neq l}\left|h_{l}\right|^{2}\left|h_{l^{\prime}}\right|^{2} E_{b}}{\sum_{l=0}^{L-1}\left|h_{l}\right|^{2} M N_{0}} .
$$

\section{Description OF THE CR TESTBED AND Results}

The aim of the CR testbed is to develop a CR network which can autoconfigure and autonomously change its parameters in a realistic environment (NLOS with multipath). For the testbed, 4 USRP B100 and 1 USRP N210 along with WBX daughterboard $(50-2200 \mathrm{MHz})$ have been distributed at our facilities in RMA (Figure 4).

In order to remotely switch on/off USRPs, a web server with power switches has been developed based on Arduino UNO and Tinkerkit relay modules (Figure 5). The Arduino and the USRP are connected to the PC by USB cables. The relay module is controlled and powered by the Arduino. 


\begin{tabular}{|c|c|c|c|}
\hline \multicolumn{2}{|c|}{ Multichannel DAA-OFDM parameters } & \multicolumn{2}{|c|}{ DADS parameters } \\
\hline FFT size & 512 & PN sequence length & 4 \\
\hline CP size & 128 & Packet length (bits) & 63040 \\
\hline $\begin{array}{l}\text { Number of modulated } \\
\text { sub-carriers }\end{array}$ & 416 & Sampling rate (Msps) & 2 \\
\hline Number of channels & 4 & Slot duration (ms) & 126.08 \\
\hline $\begin{array}{l}\text { Number of sub-carriers } \\
\text { per channel }\end{array}$ & 104 & $\begin{array}{l}\text { FDD mode } \\
\text { maximum } \\
\text { throughput (kbps) }\end{array}$ & 500.00 \\
\hline Packet length (bits) & 81920 & $\begin{array}{c}\text { TDD mode } \\
\text { maximum } \\
\text { throughput (kbps) }\end{array}$ & 250.00 \\
\hline $\begin{array}{l}\text { Constellation per sub- } \\
\text { carrier }\end{array}$ & QPSK & & \\
\hline Sampling rate (Msps) & 2 & & \\
\hline Slot duration (ms) & 126.08 & & \\
\hline $\begin{array}{l}\text { FDD mode maximum } \\
\text { throughput (kbps) }\end{array}$ & 649.75 & & \\
\hline $\begin{array}{l}\text { TDD mode maximum } \\
\text { throughput (kbps) }\end{array}$ & 324.87 & & \\
\hline
\end{tabular}

Fig. 6. Parameters chosen for the multichannel DAA-OFDM and DADS waveforms

The parameters chosen for the multichannel DAA-OFDM and DADS waveforms are given in Figure 6.

Figure 7 shows the simulated BER performance of the multichannel DAA-OFDM waveform and the DADS waveform in AWGN channels and 1-tap Rayleigh channels. One can observe that the multichannel DAA-OFDM waveform has better BER performance than the DADS waveform by $4 \mathrm{~dB}$ at BER .

Figure 8 shows the setup for the scenario. In this scenario, a jammer perturbs the data transmission between two CR nodes. The first CR node has a quad-core CPU@2.9 GHz with 8 GB RAM and the second CR node has a quad-core CPU @2.4 $\mathrm{GHz}$ with 4 GB RAM.

The CR nodes operate at $433.92 \mathrm{MHz}$. The CR nodes can use either the multichannel DAA-OFDM waveform or the DADS waveform in a TDD mode. Figure 9 shows the spectrogram of the $\mathrm{CR}$ nodes when a jammer perturbs the data transmission. At the beginning of the demonstration, the

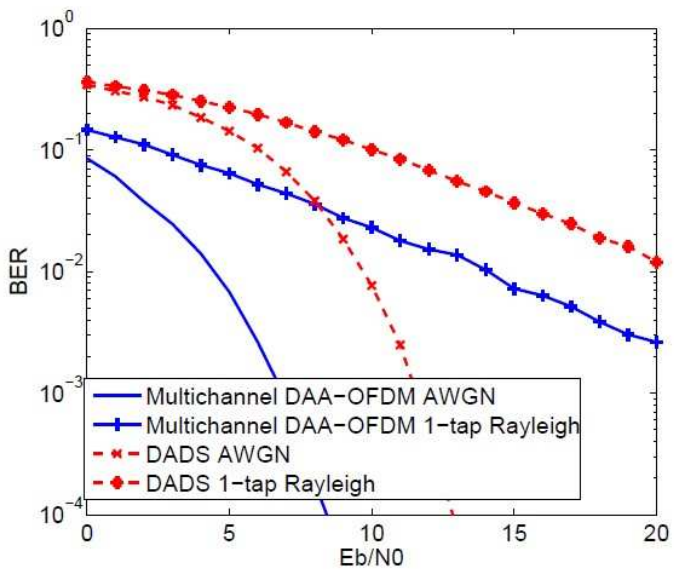

Fig. 7. BER performance of the TDD-OFDMA and TDD-DADS waveforms

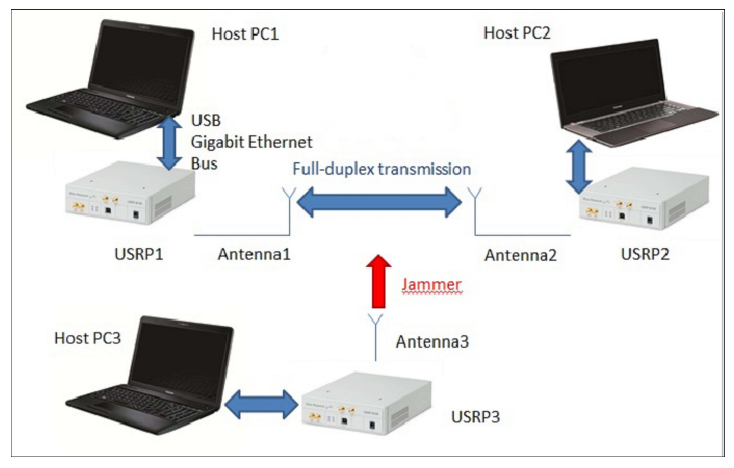

Fig. 8. Setup used for the demonstrator

CR nodes use the DADS waveform. At a certain moment, a sweeping jammer perturbs the transmission and the throughput of both users drops. When the user throughput drops under a particular threshold ( $1 \mathrm{kbps})$, the CR nodes switch from the DADS waveform to the multichannel DAA-OFDM waveform automatically while maintaining communication. The multichannel DAA-OFDM waveform adjusts dynamically the channel used for transmission and mitigates interference by sensing its operational electromagnetic environment. Therefore, whenever the jammer perturbs the channel used for transmission, the multichannel DAA-OFDM waveform is able to jump to another channel while maintaining communication.

In the case of reactive jammers, frequency hopping can be enabled by a predefined hopping sequence only known to the CR nodes. The USRP RF frontend allows to perform frequency hopping over a large frequency range from $70 \mathrm{MHz}$ to $6 \mathrm{GHz}$. Some tests with the USRP have shown that the maximum frequency tuning time is about $700 \mathrm{~ns}$, giving a maximum limit on the hop rate of 1500 hops/s.

During measurements of the multichannel DAA-OFDM and the DADS waveforms in a TDD mode, we have observed

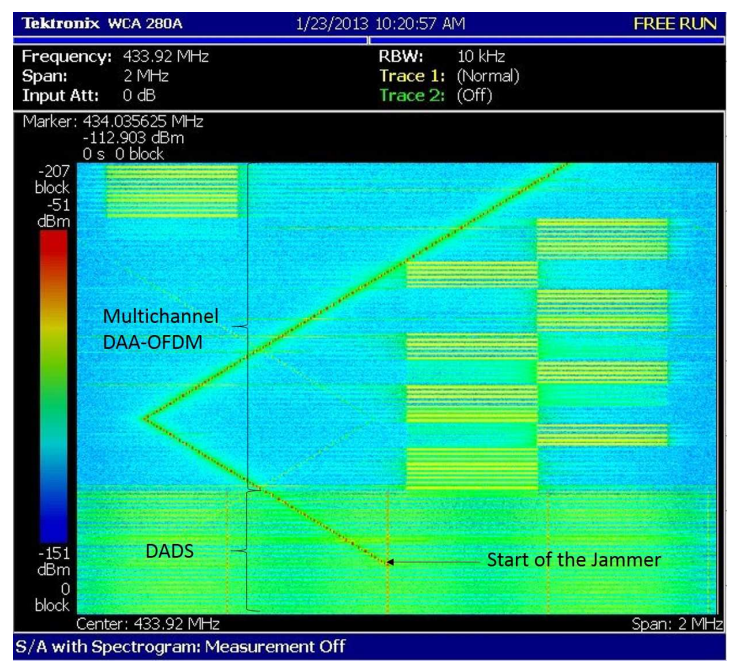

Fig. 9. Spectrogram showing the cognitive capability of the CR nodes 
that the averaged throughput corresponds to the maximum throughput $250 \mathrm{kbps}$ for the DADS waveform when streaming either from the first $\mathrm{CR}$ node to the second $\mathrm{CR}$ node or from the second $\mathrm{CR}$ node to the first CR node. However, the averaged throughput for the multichannel DAA-OFDM waveform is less than the maximum throughput $324.87 \mathrm{kbps}$ from the first CR node to the second CR node $(64 \mathrm{kbps})$ and from the second CR node to the first CR node $(160 \mathrm{kbps})$. The difference between the maximum throughput and the averaged throughput can be explained by three factors. The first factor is the processing power of the CR nodes. The second factor is the larger complexity of the receivers compared to the transmitters. The third factor is the larger complexity of the multichannel DAA-OFDM waveform compared to the DADS waveform. Due to these three factors, the two CR nodes are not able to process every packet in $252.16 \mathrm{~ms}$. At the transmit side, the same packet can be sent for several frame periods. At the receive side, some packets can be missed. Therefore, the complexity of CR waveforms is a very important issue when they are implemented on SDR platforms especially when using general purpose processors (GPP).

Figure 10 shows some throughput results for the multichannel DAA-OFDM and DADS waveforms when considering different sampling rates in a FDD mode. One can observe that the averaged throughput of the DADS waveform is close to the maximum throughput for any sampling rate. There is a small decrease in averaged throughput compared to the maximum throughput because the two CR nodes are not able to process every packet in $126.08 \mathrm{~ms}$. This small decrease does not appear in a TDD mode (averaged throughput=maximum throughput) because the processing thread has enough time to process every packet in $252.16 \mathrm{~ms}$. For high sampling rates, the averaged throughput of the multichannel DAAOFDM waveform is lower than the maximum throughput. However, for lower sampling rates, the difference between the averaged throughput and the maximum throughput decreases. Therefore, more complex waveforms such as the multichannel DAA-OFDM waveform can still be used using GPP either at lower sampling rates or by defining a frame with multiple slot periods such as in a TDD mode or in a time division multiple access (TDMA) to increase the time in which the packet can be processed.

\begin{tabular}{|c|c|c|c|c|}
\hline \multirow[b]{2}{*}{$\begin{array}{l}\text { Sampling rate } \\
\text { (Msps) }\end{array}$} & \multicolumn{2}{|c|}{$\begin{array}{l}\text { Multichannel DAA-OFDM } \\
\text { demonstration results }\end{array}$} & \multicolumn{2}{|c|}{ DADS demonstration results } \\
\hline & $\begin{array}{l}\text { FDD mode } \\
\text { maximum } \\
\text { throughput } \\
\text { (kbps) }\end{array}$ & $\begin{array}{l}\text { Averaged } \\
\text { throughput }\end{array}$ & $\begin{array}{c}\text { FDD mode } \\
\text { maximum } \\
\text { throughput } \\
(\mathrm{kbps})\end{array}$ & $\begin{array}{l}\text { Averaged } \\
\text { throughput }\end{array}$ \\
\hline 2 & 649.75 & 87.00 & 500.00 & 450.00 \\
\hline 1 & 324.88 & 90.00 & 250.00 & 231.00 \\
\hline 0.5 & 162.44 & 96.00 & 125.00 & 113.00 \\
\hline 0.25 & 81.22 & 79.00 & 62.50 & 58.00 \\
\hline
\end{tabular}

Fig. 10. Throughput results for the multichannel DAA-OFDM and DADS waveforms

\section{CONCLUSION}

This paper has provided a description of a CR testbed based on USRP platforms and the CogWave framework with its two waveforms, i.e. the multichannel DAA-OFDM waveform and the DADS waveform. CogWave is an open-source software framework aiming at developing cognitive radio (CR) waveforms. CogWave provides the means to implement different CR waveforms and to develop rules to switch between different $\mathrm{CR}$ waveforms during run-time according to the user needs and the electromagnetic environment. For a scenario in which a jammer perturbs the data transmission between two CR nodes, it has been shown that when the throughput drops under a predefined threshold, the CR nodes switch from the DADS waveform to the multichannel DAA-OFDM waveform automatically while maintaining communication. Due to the low complexity of the DADS waveform, the averaged throughput is close to the maximum throughput for sampling rates up to 2 Msps. For more complex waveforms such as the multichannel DAA-OFDM waveform, the averaged throughput is close to the maximum throughput at lower sampling rates $(0.25 \mathrm{Msps})$. Therefore, the parameters of CR waveforms should be properly adjusted to take into account the processing time vs the acquisition time when implemented on SDR platforms especially when using general purpose processors (GPP).

\section{REFERENCES}

[1] J. Mitola III, "Software radios: Survey, critical evaluation and future directions," IEEE Aerospace and Electronic Systems Magazine, vol. 8, no. 4, pp. 25-36, Apr. 1993.

[2] - "Cognitive radio: making software radios more personal," IEEE Personal Communications, vol. 6, no. 4, pp. 13-18, Aug. 1999.

[3] "Cogwave: Open-source software framework for cognitive radio waveforms." [Online]. Available: https://github.com/vlenircissrma/CogWave

[4] V. Le Nir and B. Scheers, "Implementation of an adaptive OFDMA PHY/MAC on USRP platforms for a cognitive tactical radio network," in Military Communications and Information Systems Conference (MCC'2012), Gdansk, Poland, Oct. 2012.

[5] — , "DADS with short spreading sequences for high data rate communications or improved BER performance," in Military Communications and Information Systems Conference (MCC'2013), Saint-Malo, France, Oct. 2013.

[6] "Gnu radio." [Online]. Available: http://www.gnuradio.org

[7] "Omnet++ network simulation framework." [Online]. Available: http://www.omnetpp.org

[8] "Network simulator ns-3." [Online]. Available: http://www.nsnam.org/

[9] D. Danev, E. Axell, and E. G. Larsson, "Spectrum sensing methods for detection of DVB-T signals in AWGN and fading channels," in Proceedings of the IEEE 21st International Symposium on Personal Indoor and Mobile Radio Communications (PIMRC'2010, Istanbul, Turkey, Sep. 2010.

[10] J.-J. van de Beek, M. Sandell, and P. O. Borjesson, "ML Estimation of Time and Frequency Offset in OFDM Systems," IEEE Transactions on Signal Processing, vol. 45, no. 7, pp. 1800-1805, Jul. 1997.

[11] C. N. Georghiades, "Blind Carrier Phase Acquisition for QAM constellations," IEEE Transactions on Communications, vol. 45, no. 11, pp. 1477-1486, Nov. 1997.

[12] H. Meyr, M. Moeneclaey, and S. A. Fechtel, Eds., Digital Communications Receivers: Synchronization, Channel Estimation, and Signal Processing. John Wiley and Sons, 1998. 